there needs to be an effective mechanism for transferring the results of reseach into policy. This task of informing the development of policy is complementary to that of carrying out research relevant to policy and is an essential part of a strategy of promoting evidence based policymaking.

The charitable foundations are uniquely placed not only to make this happen but to guarantee the independence of such a centre through their resources. The scale of the operation almost certainly demands collaboration between the foundations to provide the necessary infrastructure and credibility. At a time of almost unprecedented interest in health policy, the establishment of a national centre for analysing health policy calls for serious and urgent consideration. The centre would not supplant existing institutions but would work alongside them to provide a bridge between research and policy. It would be essential for the centre to build on the strengths of established programmes and to add value to these through its activities.

One way of doing this would be to set up a small group of experienced analysts in the centre, with additional input being provided by staff from existing units. This "hub and spoke" model would have the virtue of combining a central focus with a network of researchers and analysts around Britian. It would also have the virtue of addressing policy issues at multiple levels in the health sector and not concentrating solely on the national level.

Such a centre would be expected to operate across the political spectrum. Opposition parties should not be exempt from the requirement to base their policies on evidence. Margaret Beckett, Labour's shadow health minister, has inherited the bare bones of an alternative health policy from David Blunkett and will no doubt be taking this forward during the coming months. As she does so she will, hopefully, draw on published research and the results emerging from continuing studies on health policy. Although opposition parties do not enjoy the same access to the civil service's advice as ministers, there is no shortage of skill available from non-governmental agencies and research centres. It would be a missed opportunity if this advice was ignored, even if some researchers will need to be enticed out of their ivory towers to participate on the debate on policy.

One way of turning these aspirations into reality would be to create a culture in which new policies-whatever their provenance-were accompanied by a statement of the evidence that was consulted in their preparation. This might not completely prevent the promulgation of oddball ideas, but it would at least give politicians pause for thought. Evidence based policymaking needs to go hand in hand with evidence based medicine in the shaping of an NHS fit for the future.

CHRIS HAM

Director

Health Services Management Centre,

University of Birmingham,

Birmingham B15 2RT

DAVID J HUNTER Director

Nuffield Institute of Health,

University of Leeds,

Leeds LS2 9PL

RAY ROBINSON Director

Institute for Health Policy Studies,

University of Southampton,

Southampton SO17 1BJ

1 Morrison I, Smith R. The future of medicine. BMf 1994;309:1099-100

2 Robinson R, LeGrand J, eds. Evaluating the NHS reforms. London: King's Fund, 1994.

\title{
Acute dissection of the thoracic aorta
}

\section{Transoesophageal echocardiography is the investigation of choice}

Untreated patients with acute dissection of the thoracic aorta have a mortality of more than $1 \%$ per hour. ${ }^{1}$ Lowering the systolic blood pressure reduces the risk of extension, so every patient who might have a dissection should receive antihypertensive treatment while waiting for a definitive diagnosis by imaging. Such patients should be treated in a high dependency area, where the electrocardiogram can be monitored continuously. The systolic blood pressure should be kept below $110 \mathrm{~mm} \mathrm{Hg}$ by an infusion of labetalol. This is the drug of choice as it causes a rapid reduction in blood pressure that can be controlled by altering the rate of infusion.

Which diagnostic imaging technique should be used? It should provide a fast and accurate diagnosis and identify damage to the ascending aorta. Ideally, it should also show the site of the intimal tear, the distal extent of any dissection, and the presence of associated complications including tamponade, aortic regurgitation, and the involvement of the origins of the coronary arteries and aortic branches. ${ }^{2}$

Aortography has long been the standard investigation, but it has disadvantages. These include delay while the angiography team is assembled, the risk of aortic rupture during manipulation of the catheter, and the nephrotoxicity of radiological contrast media in patients whose renal function may already be compromised. Echocardiography, computed tomography, and magnetic resonance imaging all have advantages over aortography. ${ }^{23}$
Transthoracic or suprasternal echocardiography may show a dissection flap in the ascending aorta or arch. This sign is highly specific, and imaging can be performed quickly and easily in most patients. ${ }^{4}$ Unfortunately, however, the sensitivity of transthoracic echocardiography is limited to about $60 \%$, so that negative findings have to be followed by further investigation to exclude dissection.

Computed tomography enhanced with a radiological contrast medium, available in many district hospitals, is minimally invasive and its sensitivity and specificity at least equal those of aortography. ${ }^{4}$ The diagnostic accuracy of ultrafast and spinal computed tomography is even better, but these techniques are not yet widely available. Magnetic resonance imaging provides excellent images of the whole aorta. Cardiac gated and "cine" techniques give information on luminal blood flow and valvar regurgitation-at the expense of a prolonged scanning time. ${ }^{2}$

Both computed tomography and magnetic resonance imaging require the transfer of the patient to a scanner. Access to the patient is restricted during scanning, and for magnetic resonance imaging a further complication is the need to use non-ferrous and shielded monitoring equipment. At present neither technique allows real time scanning, and good cooperation is needed from the patient for high quality images. All this means that, despite their excellent diagnostic capabilities, computed tomography and magnetic resonance 
imaging have only a limited place in the management of patients with suspected acute dissection.

In experienced hands the emergency investigation of choice is transoesophageal echocardiography. ${ }^{3}$ This can be performed rapidly in the cardiac care unit, and the patient remains accessible to medical and nursing staff. ${ }^{6}$ It provides detailed anatomical information about the morphology of a dissection-including the involvement of the coronary artery ostia-and it also gives functional information about other associated complications, such as aortic regurgitation and tamponade. ${ }^{6}$ Biplane and multiplane probes have reduced the blind spot in the upper ascending aorta and causes of artefact such as calcification of the aortic wall are now well recognised. ${ }^{7}$ Transoesophageal echocardiography will also show the presence of an intramural haematoma, ${ }^{8}$ persistent flow in the false lumen, and communications between the lumens, each of which is associated with a poor long term outcome. ${ }^{9}$

Immediate surgery is mandatory when the ascending thoracic aorta is involved in the dissection (Stanford classification type $A^{10}$ ). ${ }^{2}$ This entails excision of the intimal tear, reapproximation of the dissected aortic layers, and interposition of a Dacron graft. ${ }^{11}$ When the aortic valve is involved, it is repaired by resuspending the commissures unless the patient has Marfan's syndrome, when the ascending aorta and the aortic valve are usually replaced with a composite graft. ${ }^{11}$

Attitudes about performing bypass surgery for "coincidental" coronary disease at the same time as emergency aortic surgery are changing. ${ }^{12}$ Coronary disease can be assessed fully only by coronary angiography, with its attendant risks and preoperative delay. In patients with no history of ischaemic heart disease many surgeons now operate without performing coronary angiography if transoesophageal echocardiography shows that the dissection does not affect the coronary ostia.

Aggressive control of the blood pressure and prolonged bed rest is the usual initial treatment for patients with uncomplicated dissection sparing the ascending aorta (Stanford type B) ${ }^{2}$ as emergency surgery to the descending thoracic aorta carries a substantial mortality when compared with medical treatment. Surgery should be reconsidered if there is evidence of aortic rupture, proximal extension of the dissection, or ischaemic complications. ${ }^{13}$

The optimal management of patients with suspected dissection requires close liaison between district hospitals and cardiac surgical centres and use of local guidelines for investigation that reflects the available skill. Patients with a low clinical likelihood of dissection who are in a stable cardiovascular state should undergo prompt local investigation with a nominated non-invasive technique. Unstable patients with a high likelihood of dissection should receive medical treatment and be transferred immediately to the surgical centre for both diagnostic imaging and management. If skill in transthoracic or transoesophageal echocardiography is available locally these procedures can be performed while transport is awaited, but doing so should not delay transfer. A videotaped record of the study should accompany the patient to the surgical centre, where repeat transoesophageal echocardiography can be performed in the anaesthetic room if necessary. This approach minimises delay, an essential step in lowering the mortality of acute dissection.

ADRIAN P BANNING British Heart Foundation research fellow MICHAEL S T RUTTLEY Consultant radiologist

FRANCESCO MUSUMECI

Consultant cardiac surgeon

ALAN G FRASER

University Hospital of Wales, Consultant cardiologist
1 Hirst AE, Johns VJ, Wesley Kane SW Jr. Dissecting aneurysm of the aorta: a review of 505 cases. Medicine 1958;37:217-79.

Cigarros JE, Isselbacher EM, Desanctis RW, Eagle KA. Diagnostic imaging in the evaluation of suspected thoracic aortic dissection. $N$ Engl F Med 1993;328:35-43.

3 Khandheria BK. Aortic dissection: the last frontier. Circulation 1993;87:1765-8.

4 Nienaber CA, von Kodolitsch Y, Nicolas V, Siglow V, Piepho A, Brockhoff C, et al. The diagnosis of thoracic aortic dissection by non-invasive imaging procedures. N Engl f Med 1993;328:1-9.

5 Nienaber CA, Speilmarm RP, von Kodolitsch Y, Siglow V, Piepho A, Jaup T, et al. Diagnosis of thoracic aortic dissection: magnetic resonance imaging versus transesophageal echocardiography. Circulation 1992;85:434-47.

6 Erbel R, Engberding R, Daruel W, Roelandt J, Vaser C, Rennollet H, et al. Echocardiography in diagnosis of aortic dissection. Lancet 1989;i:457-61.

7 Evangelista A, Gonzalez-Alujas T, Garda del Castillo H, Anivarro I, Salas J, Soler-Soler J. Are artefacts a major drawback for the diagnosis of ascending aortic dissection by transoesophageal echocardiography? [Abstract.] Eur Hearr f 1993;14(suppl):391.

8 Robbins RC, McManus RP, Mitchell RS, Latter DR, Moon MR, Olinger GN, et al. Management of patients with intramaural hematoma. Circulation 1993;88:1-10.

9 Erbel R, Oelert H, Meyer R, Puth M, Mohr-Katoly S, Hausman D, et al. Effect of medical and surgical therapy on aortic dissection evaluated by transesophageal echocardiography. Circulation 1993;87:1604-5.

10 Dally PO, Trueblood HW, Stinson EB, Weurfein RD, Shurnway NE. Management of acute aortic dissections. Ann Thorac Surg 1970;10:237-47.

11 Bacher RE, Termignon RL, Dreyfus G, Goudot B, Martinelli L, Piquois A, et al. Aortic dissection prevalence, cause and results of late operations. F Thorac Cardiovasc Surg 1994;108:199-206.

12 Kern MJ, Serota H, Callicoat P, Deligonul U, Lee WH, Aguirre F, et al. Use of coronary angiography in the preoperative management of patients undergoing urgent repair of the thoracic aorta. Am Heart $f$ 1990;119:143-8.

13 Dapunt OE, Galla JD, Sadegift AM, Lansman SL, Mezrow CK, de Asis Ra, et al. The natural history of thoracic aortic aneurysms. $₹$ Thorac Cardiovasc Surg 1994;107:1323-33.

\section{GPs, patients, and the distance between them}

\section{Planning would help to increase access}

The issue of coherent catchment areas for primary care is raised again by Murray and colleagues in this week's journal (p 100)..$^{1}$ The problem can be viewed from two perspectives: the distance that the patient is from the doctor and the distance that the doctor is from the patient.

For most people the convenience of the nearest surgery is the main determinant of where they register. ${ }^{2}$ Nevertheless, many go further afield, and historic factors may be important in their choice. When they move, those in urban areas often remain with the devil they know-"the desire to maintain a link with a doctor known to the family ... a appears to be stronger than any wish to minimise the distance travelled to the practice premises." 3 The availability of a woman doctor, and, for patients from ethnic minorities, doctors from their own cultural group, may also be important.

Since 1948 , when a salaried component was added to the simple equation between capitation and income, there has been little evidence that doctors retain migrants or accept allcomers for financial reasons. The majority of patients on most urban general practitioners' lists live within the practice's catchment area. This limits unnecessary travelling, facilitates care with local teams, and ensures that local populations do not lose out to more distant but mobile patients. But maintaining the right of patients to choose within limits seems a desirable option and one that services should be capable of accommodating. 